Trauma Berufskrankh 2008 · 10[Suppl 1]:47-52 DOI 10.1007/s10039-007-1217-4

Online publiziert: 21. März 2007

๑) Springer Medizin Verlag 2007

\author{
A. Jubel · J. Andermahr $\cdot$ K.E. Rehm \\ Klinik für Unfall-, Hand und Wiederherstellungschirurgie, \\ Klinikum der Universität zu Köln, Köln
}

\title{
Intramedulläre Schienung von Klavikulafrakturen
} auf hin, dass beim Erwachsenen Schmerzen und verletzungsbedingte Behinderungen durch die Fraktur innerhalb der ersten 3 Wochen nach dem Unfall häufig unterschätzt werden. Aus der in den letzten Jahren publizierten Literatur geht hervor, dass $10-30 \%$ der konservativ behandelten Patienten klinisch, radiologisch und subjektiv unbefriedigende Ergebnisse aufgrund einer Verkürzung [9, 21], einer Pseudarthrose $[12,29]$ oder einer schlechten Schulterfunktion erzielten [21].

Das Standardverfahren zur operativen Therapie von Frakturen des mittleren Klavikuladrittels stellt die Plattenosteosynthese dar [18]. Ihre besondere Problematik besteht darin, dass die Zuggurtungsseite der Klavikula und damit die optimale Lage der Platte je nach Belastungsrichtung und Rotation des Arms variieren. Eine Biegebelastung des Implantats kann durch keine Plattenlage verhindert werden. Aus diesem Grund muss, um einen Plattenbruch zu vermeiden, ein im Verhältnis zur Knochenstärke sehr groß dimensioniertes Implantat gewählt werden. Um ein derartiges Implantat, in der Regel eine schmale DCP, LCP oder eine Kleinfragmentrekonstruktionsplatte, anzubringen, ist eine sichere Schraubenverankerung erforderlich, was nicht immer gelingt. Es besteht dann die Gefahr eines Implantatausrisses. Dazu kommt ein aus- gedehnter Zugang mit zusätzlicher Ablösung von Weichteilen $[3,17,26]$. Typische Probleme der Plattenosteosynthese sind $[3,4,17,26]$ :

- häufig auftretende hypertrophe Keloidnarben,

- Implantatlockerungen,

- Pseudarthrosen und

- Refrakturen nach Implantatentfernung.

tannagels in der S-förmig gebogenen Klavikula [16], nach dem Prinzip der „elastisch stabilen intramedullären Nagelung“" (ESIN), wie sie von Métaizeau u. Prévot für die Frakturen der langen Röhrenknochen im Kindesalter beschrieben wurde. Aus biomechanischer Sicht ist die intramedulläre Lage des Implantats ideal, da so das Problem der wechselnden Zuggurtungsseite bei unterschiedlichen Belastungen der Klavikula umgangen wird.

Die erste intramedulläre Schienung einer Klavikulafraktur wurde 1907 von Albin Lambotte beschrieben [7]. Seitdem berichteten mehrere Autoren über verschiedenen Modifikationen dieser Technik [16, 19, 23, 24]. Auch Küntscher modifizierte das Verfahren durch beidseits umgebogene Enden [18]. Aufgrund der insgesamt im Vergleich zur konservativen Behandlung sehr viel höheren Komplikationsrate hat sich bisher keine dieser intramedullären Techniken durchsetzen können [28]. Gefürchtet sind v. a. die bereits mehrfach beschriebenen intrathorakalen Dislokationen der Kirschner-Drähte [5, 14, 20, 28].

Allen bisher beschriebenen Methoden ist gemeinsam, dass es sich nicht um intramedulläre Verfahren im eigentlichen Sinne handelt. Die Stabilität dieser adaptierenden Kirschner-Draht-Osteosynthesen basiert auf dem Prinzip, dass sich das Implantat jeweils in der Kortikalis des medialen und lateralen Fragments verankert. Es kann jedoch durch Bewegungen der Klavikula in der Kortikalis auslockern und nach medial oder lateral herauswandern.

Im Gegensatz dazu beruht die hier vorgestellte Technik auf einer langstreckigen intramedullären Stabilisierung durch die elastische Verspannung eines geraden Ti-

\section{Operationstechnik}

Das Operationsprinzip besteht in einer langstreckigen intramedullären Verspannung eines elastischen, vom sternalen Ende aus eingebrachten Titannagels im Markraum der Klavikula.

\section{Anästhesie und Lagerung}

Für die Operation, die in Intubationsnarkose durchgeführt wird, wird der $\mathrm{Pa}$ tient in Rückenlagerung auf einem röntgendurchlässigen Operationstisch positioniert. Um ausreichend Platz zum Durchschwenken des Bildwandlers zu haben, wird sein Kopf in den Saal gedreht und der Tisch auf der Säule so weit wie möglich in den Saal hineingefahren (lange Narkoseschläuche!). Der Anästhesist und das Narkosegerät stehen am Fuß des Patienten.

Der Arm und die Schulter der betroffenen Seite werden komplett desinfiziert und frei gelagert. Der Operateur steht bevorzugt auf der unverletzten Seite, um so wärts einbringen zu können. Der Assistent steht auf der verletzten Körperseite. den Nagel von medial nach lateral vor- 


\section{Reposition der Fraktur}

In Ergänzung zum Standardinstrumentarium des TEN-Set (Fa. Clinical House, Bochum) benutzen wir 2 kleine abgewinkelte Pfrieme zur Erweiterung des proximalen Markraums. Die Haut über dem sternalen Klavikulaende wird auf einer Länge von etwa $1 \mathrm{~cm}$ inzidiert. Der optimale Eintrittspunkt befindet sich 1-2 $\mathrm{cm}$ distal des Sternoklavikulargelenks im Zentrum der proximalen Klavikula. Die ventrale Kortikalis wird zunächst mit dem 2,5-mm-Bohrer eröffnet und anschließend mit einem um $30^{\circ}$ abgewinkelten Pfriem erweitert. Am sternalen Ende hat die Klavikula mit $2,5 \mathrm{~cm}$ den größten Durchmesser, sodass hier die Gefahr einer Perforation der Gegenkortikalis gering ist.

Ein Titannagel mit einem Durchmesser von $2,5-3,5 \mathrm{~mm}$ (nicht dünner!) wird im Handbohrfutter unter Bildwandlerkontrolle mit drehenden Bewegungen nach lateral bis zum Frakturspalt vorgetrieben, ohne die Gegenkortikalis zu perforieren. Hierbei dient die gebogene Spitze des Implantats als Kufe. Die Fraktur wird unter Bildwandlerkontrolle repositioniert. Gelingt die geschlossene Reposition nicht, wird zunächst versucht, die Fragmente mit perkutanen spitzen Repositionszangen einzurichten. Ist es auch hiermit nicht möglich das distale Fragment mit dem TEN aufzufädeln, wird eine etwa $2 \mathrm{~cm}$ lange Hilfsinzision in Höhe der Fraktur angelegt, die eine direkte Manipulation der Fragmente und der Nagelspitze ermöglicht.

Nach der Reposition wird der elastische Nagel, wiederum unter drehenden Bewegungen, ins laterale Fragment vorgetrieben. Zusätzliche Fragmente werden im Weichteilverbund belassen und locker angelagert, jedoch nicht fixiert. Nach abschließender Bildwandlerkontrolle erfolgen die Kürzung des Nagels mit einem kräftigen Seitenschneider und die Hautnaht in Intrakutaneinzelknopftechnik mit Monocryl $^{\circ}$ 4/o.

\section{Nachbehandlung}

Eine postoperative Ruhigstellung wird nicht durchgeführt. Die Patienten werden angehalten, den Arm schmerzorientiert zu bewegen und im täglichen Leben einzusetzen. Bei Klavikulafrakturen mit einem ausgesprengtem Keil oder mehrfragmentären Frakturen wird die Abduktion im Schultergelenk bis zum Abschluss der 3. postoperativen Woche auf $90^{\circ}$ limitiert. Zusätzlich wird bei Polytraumatisierten und bei Patienten mit Begleitverletzungen eine krankengymnastische Übungsbehandlung verordnet. Eine Mobilisation an Unterarmgehstützen ist möglich.

Die Metallentfernung wurde den Patienten bei radiologisch nachgewiesener Frakturheilung freigestellt, zumeist aber gewünscht.

\section{Ergebnisse der Behandlung}

\section{Prospektive Anwendungsbeobachtung}

\section{Patienten und Methode}

Von Dezember 1996-Juni 2004 wurde bei 132 Patienten mit 136 Klavikulafrakturen eine intramedulläre Osteosynthese durchgeführt. Es handelte sich um 89 Männer und 43 Frauen. Das mittlere Alter betrug 32,9 Jahre (13-74 Jahre).

Die Patienten wurden im Mittel 8 Tage (o-30 Tage) nach dem Unfallereignis operiert. Bei 78 Frakturen gelang die geschlossene Reposition. Bei 58 Frakturen musste eine zusätzliche 1-2 cm lange Hautinzision in Höhe der Fraktur angelegt werden. In den meisten Fällen konnte ein Nagel von $3 \mathrm{~mm}$ Durchmesser verwendet werden. Bei zierlichen Personen mit engem Markraum (Frauen) benutzten wir einen Nagel mit einem Durchmesser von 2,5 $\mathrm{mm}$. Nur in je 2 Fällen waren dünnere $(2 \mathrm{~mm})$ oder stärkere $(3,5 \mathrm{~mm})$ Implantate erforderlich. Die mittlere Durchleuchtungszeit betrug 2,9 min (Median 2,6 min, Spannweite $0,13-7,4 \mathrm{~min})$.

\section{Ergebnisse}

Bei Patienten mit isolierten Verletzungen des Schlüsselbeins konnte das subjektive Schmerzempfinden am Tag vor und am 3. Tag nach der Operation mit Hilfe einer visuellen Analogskala (o-10o Punkte) abgefragt werden. Vor der Operation wurden die subjektiven Schmerzen im Mittel mit $72,4 \pm 15$ Punkten (30-10o Punkte) angegeben. Am 3. postoperativen Tag wurden sie mit 18,9 \pm 10 Punkten (o-4o Punkte) signifikant niedriger bewertet $(\mathrm{p}<0,001)$.

Analog hierzu wurde eine signifikante Verbesserung der Beweglichkeit des verletzten Schultergelenks festgestellt. Die Abduktion im Schultergelenk war präoperativ im Mittel bis $42^{\circ}\left(30-70^{\circ}\right)$ und postoperativ bis $121^{\circ}\left(80-180^{\circ}\right)$ möglich $(\mathrm{p}<\mathrm{O}, \mathrm{OO} 1)$.

Das Implantat wurde im Mittel nach 7,6 Monaten (2-24 Monate) entfernt.

Mit Hilfe des Constant-Scores [6] zur Beurteilung der Funktion des Schultergelenks konnte bei 106 Patienten das klinische Ergebnis 12 Monate nach der Metallentfernung evaluiert werden. Der mittlere Wert des Constant-Scores betrug 97,1 $\pm 3,5$ von 100 maximal erreichbaren Punkten. Bei allen Patienten war die Beweglichkeit des Schultergelenks frei. Kammgriff und Schürzengriff konnten von allen Patienten vorgeführt werden.

Bei 8 Patienten mussten wir 1-33 Wochen nach dem Ersteingriff den an der Einschlagstelle zu langen Nagel kürzen, da es dort zu Schmerzen und Hautreizungen gekommen war.

Bei 3 Patienten mit einer mehrfragmentären Fraktur sahen wir radiologisch eine sekundäre Verkürzung der Klavikula von $1,5 \pm 0,5 \mathrm{~cm}$. Bei 5 Patienten wurde eine laterale Perforation beobachtet. In 1 dieser Fälle kam es nach 2 Monaten zur Wanderung des Implantats in die lateralen Weichteile, sodass der Nagel über eine laterale Inzision vorzeitig entfernt wurde. Bei 3 Patienten wurde eine temporäre neurologische Symptomatik in Form von Kribbelparästhesien des betroffenen Arms und der Hand beobachtet. Bei einer polytraumatisierten Patientin trat eine Pseudarthrose auf.

\section{Resümee}

Die Reposition der Fraktur und die Herstellung einer elastischen Stabilität am Frakturspalt sind Voraussetzung für eine rasche postoperative Schmerzfreiheit und Beweglichkeit der verletzten Schulter. Die Länge der Klavikula kann mit der gewählten Methode wiederhergestellt werden. 


\section{Prospektiv vergleichende Untersuchung}

\section{Patienten}

In diese Untersuchung wurden 53 Patienten aufgenommen, die eine Klavikulafraktur erlitten hatten und folgende Einschlusskriterien erfüllten:

- Die Fraktur war im mittleren Drittel der Klavikula lokalisiert (Typ AllmanI [1]).

- Es handelte sich um einen Typ A oder B der OTA-Klassifikation [2] am ausgewachsenen Skelett.

- Es lag eine schriftliche Einverständniserklärung der Patienten vor.

Die Patienten konnten nach einer entsprechenden Aufklärung zwischen einer konservativen und einer operativen Behandlung frei wählen. Insgesamt entschieden sich 27 Patienten für die nichtoperative und 26 Patienten für die operative Therapie.

\section{Ergebnisse}

Sie wurden bei allen Patienten vom Tag der Zustimmung zur Studie an prospektiv erfasst. Klinische Kontrollen erfolgten an Tag 3, 7, 14, 21 und 42 sowie nach 3 und 6 Monaten. An diesen Zeitpunkten wurden erhoben:

- die subjektive Einschätzung der Schmerzstärke auf einer visuellen Analogskala (VAS o-10o Punkte),

- die subjektive Zufriedenheit mit der persönlichen Situation nach Schulnoten von 1-6,

- die Dauer der Arbeitsunfähigkeit und

- der Constant-Score [6].

Die Längendifferenz wurde vom Zentrum des Jugulums bis zur Akromionspitze [16] gemessen und diente als Maß für die Klavikulaverkürzung. Die aktive Beweglichkeit der verletzten Schulter nach der Neutral-o-Methode, der DASH-Score [19] sowie unerwünschte Ereignisse, Probleme und Komplikationen wurden ebenfalls dokumentiert. Die Einschätzung des ästhetischen Ergebnisses durch den Patienten bezüglich Kalluswulst, Schulterasymmetrie, Muskelatrophie und ggf. Operationsnarbe erfolgte in Form von Schulnoten nach 6 Monaten.

Trauma Berufskrankh 2008 · 10[Suppl 1]:47-52 DOI 10.1007/s10039-007-1217-4

(C) Springer Medizin Verlag 2007

\section{A. Jubel · J. Andermahr · K.E. Rehm Intramedulläre Schienung von Klavikulafrakturen}

\section{Zusammenfassung}

Trotz der günstigen Prognose weisen 10 $30 \%$ der konservativ behandelten Patienten mit Klavikulafraktur unbefriedigende Ergebnisse auf. Die vorgestellte Technik der intramedullären Osteosynthese beruht auf einer langstreckigen intramedullären Stabilisierung durch die elastische Verspannung eines geraden Titannagels in der S-förmig gebogenen Klavikula nach dem Prinzip der „elastisch stabilen intramedullären Nagelung". Bei mit dieser Methode versorgten 132 Patienten (136 Klavikulafrakturen) zeigte sich, dass die Reposition der Fraktur und die Herstellung einer elastischen Stabilität am Frakturspalt Voraussetzung für eine rasche postoperative Schmerzfreiheit und Beweglichkeit der verletzten Schulter sind. In einer prospektiv vergleichenden Untersuchung konnten 53 Pati- enten mit Klavikulafraktur frei zwischen konservativer und operativer Behandlung wählen (27 konservativ/26 operativ). Hinsichtlich Schmerzen, subjektiver Zufriedenheit, ästhetischem und funktionellem Ergebnis, Arbeitsunfähigkeit, Längendifferenz, Constant- und DASH-Score waren die Resultate nach Operation signifikant besser als nach konservativer Behandlung. Die vorgestellte Methode ist somit bei Klavikulafrakturen des mittleren Drittels der Typen A und B der OTA-Klassifikation am ausgewachsenen Skelett indiziert.

\section{Schlüsselwörter}

Klavikulafraktur · Konservative Behandlung · Intramedulläre Osteosynthese · Elastisch

stabile intramedulläre Nagelung .

Behandlungsergebnis

\section{Intramedullary splinting of clavicular fractures}

\section{Abstract}

Despite the favourable prognosis,the results of conservative treatment for clavicular fractures are unsatisfactory in $10-20 \%$ of cases. The intramedullary fixation technique presented in this paper is based on stabilization of a lengthy section of bone by means of elastic bracing of a straight titanium nail in the S-shaped clavicula according to the principle of elastically stable intramedullary nailing. In 132 patients ( 136 clavicular fractures) treated by this method, it became obvious that realignment of the fracture and achievement of elastic stability at the fracture gap are essential for rapid freedom from pain postoperatively and for mobility of the injured shoulder. In a prospective comparative study 53 patients with clavicular frac- tures were allowed to choose between conservative and operative treatment (27 conservative/26 operative). In terms of pain, subjective satisfaction, aesthetic and functional resul ts, unfitness for work, differences in length and Constant and DASH scores the results were significantly better after surgery than after conservative treatment. The method presented is therefore indicated for type $A$ and $B$ clavicular fractures of the middle third (OTA Classification) in the mature skeleton.

\section{Keywords}

Clavicular fracture - Conservative treatment . Intramedullary osteosynthesis . Elastically stable intramedullary nailing . Treatment outcome 

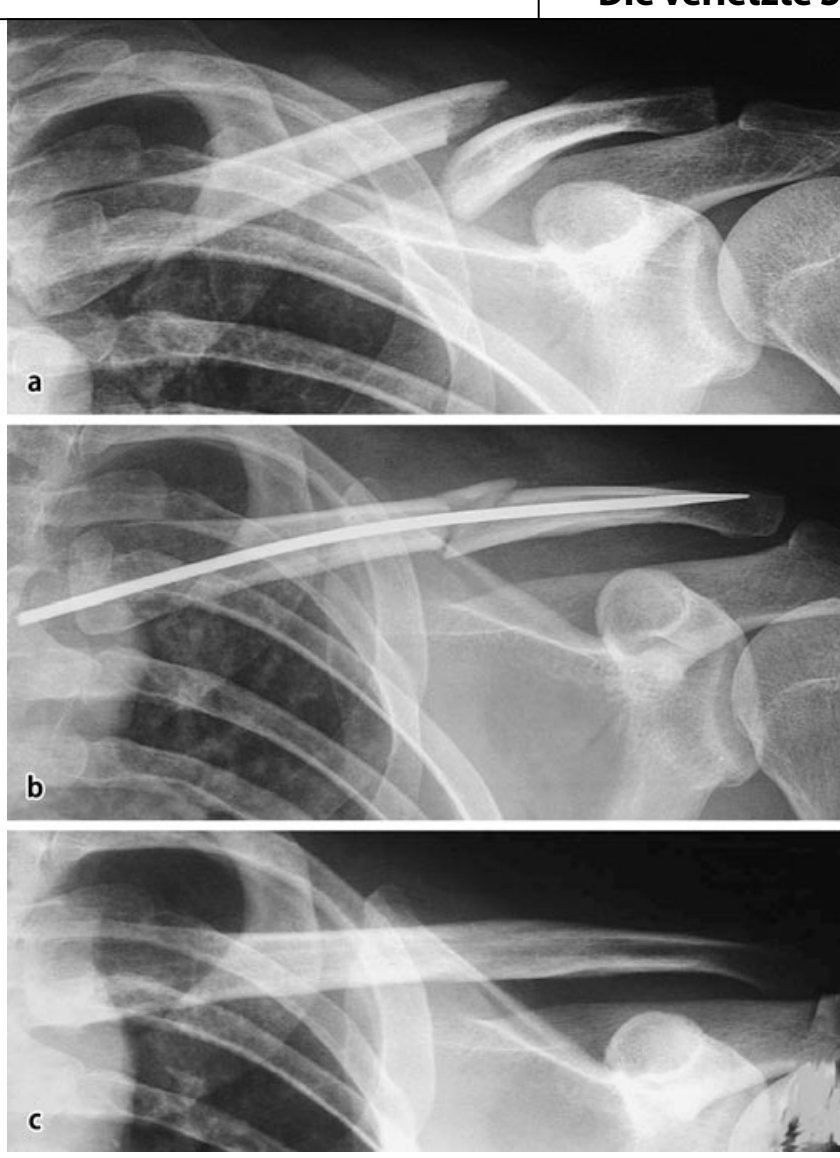
plantats, jetzt zusätz-

Digitale Röntgenaufnahmen mit hängendem Arm und Maßband wurden zur Erstuntersuchung, unmittelbar postoperativ, nach 6 Wochen, 3 Monaten und 6 Monaten angefertigt. In $\bullet$ Abb. 1 ist beispielhaft der Verlauf einer Klavikulafraktur bei einem 32-jährigen Mann, der mit dem Fahrrad gestürzt war, gezeigt.

Schmerzen. Die von den Patienten zum Zeitpunkt der Behandlungsaufnahme auf einer visuellen Analogskala (o-10o Punkte) subjektiv quantifizierten Schmerzen waren mit $77 \pm 13$ Punkten in der konservativen Gruppe und $74 \pm 18$ Punkten in der Gruppe der operierten Patienten nicht signifikant unterschiedlich. In der operativen Gruppe wurden vom Zeitpunkt der ersten Nachuntersuchung an bis zum 6 . Monat signifikant $(\mathrm{p}<0,05)$ niedrigere Schmerzwerte angegeben.

Subjektive Zufriedenheit. $\mathrm{Zu}$ Beginn der Behandlung bestand in beiden Behandlungsgruppen eine gleich große „Unzufriedenheit", die im Mittel mit einer Schulnote von 5,6 bewertet wurde. Ab dem Tag der ersten Nachuntersuchung
Abb. $1 \varangle 32$-jähriger Mann mit Klavikulafraktur nach Fahrradsturz, a präoperative Röntgenaufnahme: dislozierte Fraktur des mittleren Klavikuladrittels vom Typ A2 der OTA-Klassifikation, $\mathbf{b}$ Röntgenkontrolle am 3. postoperativen Tag: Repositionsergebnis und korrekte intramedulläre Lage des Imlicher Keil am kranialen Rand der Fraktur erkennbar, aufgrund des kaudal kortikalen Kontakts der beiden Hauptfragmente kein „Teleskopeffekt" zu erwarten, Nagel am proximalen Ende nicht umgebogen, c Ausheilungsbild nach Metallentfernung 6 Monate nach Osteosynthese: anatomisch wiederhergestellte Klavikula ohne Kalluswulst

Längendifferenz der Klavikula. Zu Beginn der Behandlung bestand in beiden Therapiegruppen eine mittlere Verkürzung der jugulo-akromialen Länge von $1,2 \mathrm{~cm}$. 6 Monate nach dem Unfallereignis wurde bei insgesamt 22 Patienten noch eine Verkürzung von $1 \mathrm{~cm}$ oder mehr gemessen. Eine Verlängerung dieser Strecke wurde in keinem Fall beobachtet. Die mittlere Verkürzung war in der Gruppe der konservativ behandelten Patienten mit 1,4 $\pm 0,9 \mathrm{~cm}$ signifikant ( $\mathrm{p}<0,001)$ größer als in der Gruppe der operierten Patienten mit $0,2 \pm 0,3 \mathrm{~cm}$.

\section{Funktionelles Ergebnis, Constant- und} DASH-Score. Zum Zeitpunkt o bestand zwischen den Therapiegruppen kein signifikanter Unterschied der Schulterbeweglichkeit für Anteversion und Abduktion, im Constant- und im DASH-Score. Während des gesamten Beobachtungszeitraums wurden in der Gruppe der operierten Patienten signifikant $(\mathrm{p}<\mathrm{0}, 001)$ bessere Werte für die Abduktion, die Anteversion $(\mathrm{p}<0,05)$, im Constant-Score $(\mathrm{p}<\mathrm{o}, \mathrm{oo1})$ und ab dem 7 . Tag im DASHScore $(\mathrm{p}<0,001)$ gemessen. Die Differenzen nahmen im zeitlichen Verlauf ab.

erteten die operierten Patienten Situation signifikant $(\mathrm{p}<0,001)$ besser als die im Rucksackverband behandelten $\mathrm{Pa}$ tienten.

Ästhetisches Ergebnis. Für die konservative Behandlung wurde bei der 6-Monats-Untersuchung im Mittel eine Note von $2,3 \pm 0,8$ vergeben, während die operierten Patienten das kosmetische Ergebnis im Mittel mit einer Note von 1,2 $\pm 0,3$ ( $\mathrm{p}<0,001)$ bewerteten. Die 9 Patienten, bei denen eine offene Reposition erforderlich war, bewerteten das kosmetische Ergebnis mit 1,2 $\pm 0,3$ nicht signifikant $(\mathrm{p}=0,594)$ schlechter als die 17 Patienten nach geschlossener Reposition mit $1,1 \pm 0,3$.

Arbeitsunfähigkeit. Für 17 der konservativ behandelten und 22 der operierten Patienten wurden Arbeitsunfähigkeitsbescheinigungen ausgestellt. Die Dauer der Arbeitsunfähigkeit war für die nichtoperierten Patienten mit 40 \pm 19 Tagen signifikant $(\mathrm{p}<0,001)$ länger als für die operierten Patienten mit $16 \pm 12$ Tagen.
Probleme und Komplikationen. Bei 4 Patienten nach konservativer Behandlung, die auch nach 6 Monaten noch über gelegentliche Missempfindungen der oberen Extremität klagten, konnte eine vermehrte Kallusformation als Ursache festgestellt werden. Die schmerzhaften und teilweise übel riechenden Hautmazerationen in der Axilla verheilten folgenlos nach Entfernung des Rucksackverbands.

Das häufigste Problem der operativen Behandlung bestand in den schmerzhaften medialen Nagelenden. Bei 2 von 7 Patienten musste dieses deshalb nach der 6. Woche in Lokalanästhesie gekürzt werden.

Bei 2 Patienten, bei denen der Titannagel die dorsolaterale Klavikula perforierte, erfolgte nach 14 und 16 Wochen die vorzeitige Implantatentfernung, ohne dass es zu einer sekundären Dislokation kam. Bei 1 dieser Patienten trat bereits in der ersten Woche nach der Primäroperation aufgrund der lateralen Perforation ein Repositionsverlust von $1 \mathrm{~cm}$ auf. 
Im Vergleich zum Ausgangsbefund (Tag o) traten bei 13 der konservativ behandelten Patienten im weiteren Verlauf Repositionsverluste auf. Ein Zusammenhang zwischen der Frakturform und dem Ausmaß des Repositionsverlusts konnte nicht festgestellt werden.

Die Dauer des stationären Aufenthalts der operierten Patienten betrug

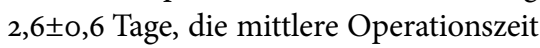
$48 \pm 17 \mathrm{~min}$.

\section{Diskussion}

Die Indikationen zur operativen Behandlung einer Klavikulafraktur und die Frage, ob hiermit ein Vorteil für die betroffenen Patienten verbunden ist, werden in der Literatur kontrovers diskutiert $[3,4,11]$. Im Vergleich zur konservativen Behandlung sind offene Verfahren wie die Plattenosteosynthese oder die offene „Markdrahtung" mit einer erhöhten Komplikationsrate verbunden und führen funktionell nicht zu besseren Ergebnissen als die nichtoperative Behandlung [4, 5, 11, 28]. Eine weit verbreitete Methode der konservativen Therapie ist die Anlage eines Rucksackverbands für die Dauer von 46 Wochen [17].

In der Vergangenheit wurde bereits von mehreren Autoren darauf hingewiesen, dass die konservative Behandlung nicht immer unproblematisch ist $[12,26$, 27]. Rowe [26] stellte fest, dass die Verletzung beim Erwachsenen bezüglich der Schmerzen und der verletzungsbedingten Behinderung innerhalb der ersten 3 Wochen nach dem Unfall häufig unterschätzt wird. Diese Feststellung kann durch die Ergebnisse unserer Arbeitsgruppe bestätigt werden.

Bei den operierten Patienten wurde durch die Stabilisierung der Fraktur eine signifikante Schmerzreduktion sowohl in Bezug auf die präoperative Situation als auch im Vergleich zu den konservativ behandelten Patienten erreicht. Aufgrund der geringeren Schmerzen sind die operierten Patienten sehr viel früher in der Lage, den Arm im verletzten Schultergelenk wieder über die Horizontale zu heben und im täglichen Leben einsetzen. Das bessere funktionelle Ergebnis zeigte sich sowohl in der besseren Schulterbe- weglichkeit als auch in den besseren Werten des Constant- und des DASH-Scores.

Mullick [20] konnte an Röntgenbildserien zeigen, dass das Ziel einer Reposition und Retention im Rucksackverband nahezu nie erreicht wird und in einigen Fällen die Dislokation sogar zunimmt. Diese Beobachtung konnte in dieser Untersuchung bestätigt werden. Bei 13 Patienten kam es im Vergleich zur Ausgangssituation im zeitlichen Verlauf zu einer Verschlimmerung der Verkürzungsfehlstellung. Ähnliche Ergebnisse fand Gäbler et al. [10] bei 165 Patienten mit Klavikulafrakturen vom Typ Allman-I, die im Rucksackverband behandelt wurden. Petracic [23] wies nach, dass bei zunehmender Spannung des Rucksackverbands eine Venenstauung des Arms noch vor einer Reposition der Fraktur auftritt. Er sah in dieser Therapieform eine „symbolische Handlung, um die Bedürfnisse des Patienten nach einem dekorativen Verband $z u$ befriedigen". Fowler [9] bemerkte bereits 1968, dass der Rucksackverband durch Scheuern und Druck in der Axilla mehr Schweirigkeiten bereiten kann als die Fraktur an sich. Zu den häufigsten Problemen der konservativen Behandlung gehören temporäre Kribbelparästhesien der Arme [22], die in der Regel nach der Lockerung des Rucksackverbands wieder verschwinden.

Unter Berücksichtigung der hohen Komplikationsrate des Rucksackverbands sollten alternative Konzepte der nichtoperativen Behandlung diskutiert werden. Hierzu gehören die rein funktionelle Behandlung ohne Verband oder die Anlage einer einfachen Armschlinge. Petracic [23] konnte in einer prospektiv randomisierten Untersuchung zeigen, dass die funktionellen, kosmetischen und radiologischen Ergebnisse nach Rucksackverband oder einfacher Armschlinge gleich sind. Patienten im Rucksackverband beklagten jedoch signifikant häufiger Probleme und Komplikationen in Form von Schmerzen, Hautproblemen, Armschwellungen und Kribbelparästhesien. Hofwijk u. van der Werken [13] verglichen bei 152 Patienten die Behandlung mit einem Rucksackverband vs. mit einer Mitella. Sie schlossen aus ihren Ergebnissen, dass der Mitella unabhängig von der Frakturdislokation der Vorzug zu geben sei, da bei gleichen funktionellen Ergebnissen die
Patientenzufriedenheit größer und die Komplikationsrate geringer waren.

Grassi et al. [11] konnten in einer retrospektiv vergleichenden Untersuchung nach einer mittleren Beobachtungszeit von 63 Monaten keine funktionellen Vorteile bei Patienten nach einer offenen Markdrahtung der Klavikula im Vergleich zur konservativ im Rucksackverband behandelten Kontrollgruppe feststellen. Eine Verkürzung von $\geq 1 \mathrm{~cm}$ hatten 4 von 40 operierten Patienten [11]. Im Gegensatz zu der hier angewandten Technik mit elastischen 3,0-3,5 mm starken Titannägeln, die von einem frakturfernen Zugang aus eingebracht wurden, verwendeten Grassi et al. [11] 2,5-mm-Kirschner-Drähte, die über die freigelegte Fraktur in der Klavikula platziert wurden. Zusätzlich wurden die operierten Patienten postoperativ im Mittel für 33 Tage in einem immobilisierenden Verband behandelt, während die in dieser Untersuchung angewandte operative Technik eine funktionelle Nachbehandlung erlaubte.

Die Angaben zur Pseudarthroserate unter konservativer Therapie in der Literatur weisen mit $0,3-15 \%$ eine große Schwankungsbreite auf [12, 25, 26, 29]. In einer aktuellen prospektiven Kohortenuntersuchung bei 581 Patienten mit diaphysären Klavikulafrakturen wurde in 4,5\% der Fälle bis zur 24. Woche keine knöcherne Heilung festgestellt [25]. Die Pseudarthroserate nach operativer Korrektur wird in der Literatur mit $4-5 \%$ in vielen Arbeiten höher eingeschätzt als nach konservativer Behandlung [17, 26]. Dies konnte durch die hier vorgestellten Ergebnisse nicht bestätigt werden. Die vergleichende Untersuchung zeigte, dass in der konservativ behandelten Gruppe 2 von 27 Klavikulafrakturen im Beobachtungszeitraum von 6 Monaten nicht ausheilten, während dies in der operativ behandelten Gruppe bei allen Frakturen der Fall war. In der inzwischen abgeschlossenen Anwendungsbeobachtung dieser Arbeitsgruppe der Jahre 1996-2004 bei 132 Patienten mit Klavikulafrakturen vom Typ Allman-I trat 1 Pseudarthrose nach ESIN auf [24].

Ein typisches Problem der elastisch stabilen intramedullären Osteosynthese besteht in einem schmerzhaften medialen Nagelende [15], welches aus diesem Grund bei 2 von 26 Patienten in der 
vergleichenden Studie nachgekürzt werden musste. Untersuchungen an Leichenpräparaten zeigten, dass die Kortikalis der Klavikula am Eintrittspunkt des Nagels sehr dünn ist und das unter Spannung stehende Nagelende dort „durchschneiden" kann.

Unter Berücksichtigung der ökonomischen Aspekte ist die operative Behandlung mit den Nachteilen der Operationskosten belastet. Bezieht man allerdings die erhebliche Verkürzung der Dauer der Arbeitsunfähigkeit mit ein, stellt sich die operative Therapie volkswirtschaftlich in einem sehr viel günstigeren Licht dar.

Für den Patienten ist der operative Eingriff mit einem 2- bis 3-tägigen stationären Aufenthalt und dem allgemeinen Risiko einer „Vollnarkose“ verbunden. Obwohl der Eingriff an der Klinik der Autoren inzwischen auch ambulant angeboten wird, nutzen dies nur wenige Patienten $(<5 \%)$.

\section{Fazit}

Die Vorteile des minimalinvasiven, wenig belastenden Eingriffs bestehen in einer raschen Schmerzlinderung, der Belastungsstabilität, der Möglichkeit einer exakten Reposition der Klavikula sowie der Wiederherstellung der Symmetrie des Schultergürtels. Es konnte gezeigt werden, dass es sich um eine sichere Methode handelt, die zu einer raschen Schmerzlinderung und frühen Belastungsfähigkeit der verletzten Schulter führt. Das Verfahren geht mit einer geringen Pseudarthroserate einher. Im Vergleich zur konservativen Behandlung wird langfristig ein besseres funktionelles und ästhetisches Ergebnis erreicht. Die Indikationen zur Anwendung der Technik bestehen bei Klavikulafrakturen des mittleren Drittels der Typen A und B der OTA-Klassifikation [2] am ausgewachsenen Skelett. Die Kontraindikationen umfassen Frakturen die älter als 3 Wochen sind, Frakturen des Typs C der OTA-Klassifikation [2] sowie eine Osteoporose der Klavikula.

\section{Korrespondierender Autor}

\section{A. Jubel}

Klinik für Unfall-, Hand und Wiederherstellungschirurgie, Klinikum der Universität zu Köln, Josef-Stelzmann-Straße 9, 50924 Köln axel.jubel@uni-koeln.de

Interessenkonflikt. Es besteht kein Interessenkonflikt. Der korrespondierende Autor versichert, dass keine Verbindungen mit einer Firma, deren Produkt in dem Artikel genannt ist, oder einer Firma, die ein Konkurrenzprodukt vertreibt, bestehen. Die Präsentation des Themas ist unabhängig und die Darstellung der Inhalte produktneutral.

\section{Literatur}

1. Allman FL Jr (1967) Fractures and ligamentous injuries of the clavicle and its articulation. J Bone Joint Surg Am 49: 774-784

2. Anonymus (1996) Fracture and dislocation compendium. Orthopaedic Trauma Association Committee for Coding and Classification. J Orthop Trauma 10: 1-154

3. AO Journal Club Eftl (2004) Clavicular midshaft fractures. Orthop Trauma Direct 2: 19-24

4. Bostman O, Manninen M, Pihlajamaki H (1997) Complications of plate fixation in fresh displaced midclavicular fractures. J Trauma 43: 778-783

5. Chu CM, Wang SJ, Lin LC (2002) Fixation of midthird clavicular fractures with knowles pins: $78 \mathrm{pa}-$ tients followed for 2-7 years. Acta Orthop Scand 73: 134-139

6. Constant CR (1987) A clinical method of functional assesment of the shoulder. Clin Orthop 214: 160164

7. Diez HG, Schmittenbecher PP, Illing P (1997) Historische Betrachtung der intramedullären Osteosynthese. In: Diez HG, Schmittenbecher PP, IIling $\mathrm{P}$ (Hrsg) Die intramedulläre Osteosynthese im Wachstumsalter. Urban \& Schwarzenberg, München Wien Baltimore

8. Eskola A, Vainionpaa S, Myllynen P et al. (1986) Outcome of clavicular fracture in 89 patients. Arch Orthop Trauma Surg 105: 337-338

9. Fowler AW (1968) Treatment of fractured clavicle. Lancet 1: 46-47

10. Gaebler C, Matis N, Neophytou I et al. (2001) Der Tornisterverband zur Reposition und Retention der Schlüsselbeinfraktur im mittleren Drittel. Osteosynthese Int 9: 146-150

11. Grassi FA, Tajana MS, D'Angelo F (2001) Management of midclavicular fractures: comparison between nonoperative treatment and open intramedullary fixation in 80 patients. J Trauma 50: 1096-1100

12. Hill JM, McGuire MH, Crosby LA (1997) Closed treatment of displaced middle-third fractures of the clavicle gives poor results. J Bone Joint Surg $\mathrm{Br}$ 79B: 537-539

13. Hoofwijk AG, van der Werken C (1988) [Conservative treatment of clavicular fractures]. Z Unfallchir Versicherungsmed Berufskrankh 81: 151-156

14. Jubel A, Prokop A, Kress J et al. (1998) Die elastisch stabile Marknagelung der Klavikulafraktur im mittleren Drittel. Hefte Unfallchir 272: 738-739

15. Jubel A, Andermahr J, Schiffer G et al. (2002) The technique of elastic-stable intramedullary nailing of midclavicular fractures. Unfallchirurg 105: 511516
16. Jubel A, Andermahr J, Schiffer G et al. (2003) Elastic stable intramedullary nailing of midclavicular fractures with a titanium nail. Clin Orthop 408: 279-285.

17. Klonz A, Hockertz T, Reilmann H (2002) [Clavicular fractures]. Chirurg 73: 90-101

18. Kuner EH, Schlickewei W, Mydla F (1982) [Surgical therapy of clavicular fractures, indications, technic, results]. Hefte Unfallheilkd 160: 76-83

19. MacDermid JC, Richards RS, Donner A et al. (2000) Responsiveness of the short form-36, disability of the arm, shoulder, and hand questionnaire, patient-rated wrist evaluation, and physical impairment measurements in evaluating recovery after a distal radius fracture. J Hand Surg [Am] 25: 330340

20. Mullick S (1967) Treatment of midclavicular fractures. Lancet 1: 499

21. Nordqvist A, Petersson C (1994) The incidence of fractures of the clavicle. Clin Orthop Relat Res 300: 127-132

22. Nordqvist A, Petersson CJ, Redlund-Johnell I (1998) Mid-clavicle fractures in adults: end result study after conservative treatment. J Orthop Trauma 12: 572-576

23. Petracic B (1983) [Efficiency of a rucksack bandage in the treatment of clavicle fractures]. Unfallchirurgie 9: 41-43

24. Rehm KE, Andermahr J, Jubel A (2004) Intramedullary nailing of midclavicular fractures with an elastic titanium nail. Operat Orthop Traumatol 16: 365-379

25. Robinson CM, Court-Brown CM, McQueen MM et al. (2004) Estimating the risk of nonunion following nonoperative treatment of a clavicular fracture. J Bone Joint Surg Am 86-A: 1359-1365

26. Rowe CR (1968) An atlas of anatomy and treatment of midclavicular fractures. Clin Orthop 58: 29-42

27. Schmit-Neuerburg KP, Weiss H (1982) [Conservative therapy and treatment results in clavicular fractures]. Hefte Unfallheilkd 160: 55-75

28. Schwarz N, Leixnering M (1984) [Failures of clavicular intramedullary wire fixation and their causes]. Aktuelle Traumatol 14: 159-163

29. White RR, Anson PS, Kristiansen T et al. (1989) Adult clavicle fractures: relationship between mechanism of injury and healing. Orthop Trans 13: 514-515 